05. 1;06.5;10

\title{
Нелинейный фотоакустический отклик на механические напряжения вблизи отверстия в пластине из алюминиевого сплава Д16
}

\author{
(С А.Л. Глазов ${ }^{1}$, Н.Ф. Морозов ${ }^{2,3}$, К.Л. Муратиков ${ }^{1}$ \\ ${ }^{1}$ Физико-технический институт им. А.Ф. Иоффер РАН, Санкт-Петербург, Россия \\ ${ }^{2}$ Институт проблем машиноведения РАН, Санкт-Петербург, Россия \\ ${ }^{3}$ Санкт-Петербургский государственный университет, Санкт-Петербург, Россия \\ E-mail: glazov.holo@mail.ioffe.ru
}

Поступило в Редакцию 6 ноября 2019г.

В окончательной редакции 6 ноября 2019г.

Принято к публикации 12 ноября 2019 г.

\begin{abstract}
Исследованы особенности поведения фотоакустического сигнала в зависимости от механических напряжений вблизи отверстия в пластине из алюминиевого сплава Д16. Обнаружен новый нелинейный фотоакустический эффект, заключающийся в появлении нелинейной составляющей фотоакустического сигнала в зависимости от механических напряжений вблизи отверстия. Данная зависимость регистрируется в сигнале на основной частоте модуляции лазерного излучения. Предложено теоретическое описание обнаруженного эффекта генерации звука по термоупругому механизму в напряженных металлах на основе учета теплового воздействия лазерного излучения на их электронную подсистему.
\end{abstract}

Ключевые слова: фотоакустическая микроскопия, механические напряжения, термоупругость, металлы, задача Кирша.

DOI: 10.21883/PJTF.2020.04.49045.18100

Термоупругая генерация звука является основой лазерных ультразвуковых и фотоакустических (ФА) методов диагностики разнообразных структур и материалов. Интерес к методикам подобного рода обусловлен их универсальностью и возможностью применения в различных частотных диапазонах и на различных пространственных масштабах. С расширением области применения таких методов представляется актуальным изучение особенностей ФА-эффектов при разных условиях, в частности при наличии в материалах внутренних механических напряжений.

К настоящему времени продемонстрирована чувствительность лазерных ФА-сигналов к дефектам и механическим напряжениям в керамиках [1-3] и металлах [4-7] различных типов. Было установлено соответствие особенностей поведения лазерных ФА-сигналов от керамических образцов с внутренними напряжениями и теоретических результатов, полученных в рамках стандартной линейной термоупругой модели формирования ФА-сигналов. Объяснение аналогичных результатов для металлов потребовало модификации классической термоупругой модели $[8,9]$ с учетом теплового воздействия лазерного излучения на электронную подсистему $[10,11]$. В рамках модифицированной линейной термоупругой модели удалось объяснить основные особенности поведения ФА-сигнала в модельных экспериментах вблизи отверстий при наличии в металлических образцах механических напряжений. Вместе с тем наряду $\mathrm{c}$ линейной связью ФА-сигналов с напряжениями вблизи отверстия наблюдался нелинейный эффект. Задачей настоящей работы является объяснение наблюдаемых нелинейных эффектов в рамках предложенной нами ранее физической модели.

В работах $[10,11]$ было показано, что амплитуда акустических колебаний, генерируемых лазерным излучением по термоупругому механизму, аномальным образом зависит от механических напряжений в металлических образцах. В [11] была предложена модель в линейном приближении, описывающая такую аномалию. Модель основана на тепловом возбуждении связанных электронов различных дефектов и неоднородностей, присутствующих в реальных металлах. В рамках предложенной модели в линейном приближении удалось объяснить особенности поведения ФА-сигнала вблизи отверстия в образцах из сплава алюминия Д16 при наличии в них одноосных напряжений, характеризующихся угловой зависимостью $\cos (2 \vartheta)$. Вместе с тем в этих же экспериментах наряду с указанной зависимостью надежно наблюдалась и существенно меньшая по амплитуде зависимость типа $\cos (4 \vartheta)$, соответствующая нелинейной связи ФА-сигнала и механического напряжения.

В соответствии с задачей Кирша распределение напряжений вокруг отверстия при одноосном напряжении определяется выражением [12,13]:

$$
\sigma(r, \vartheta)=\sigma_{x}\left(1-2 a^{2} \cos (2 \vartheta) / r^{2}\right),
$$

где $\sigma_{x}$ - одноосное напряжение вдали от отверстия, $a$ - радиус отверстия. Для учета вклада нелинейных 




Рис. 1. Двумерное распределение нелинейной составляющей ФА-сигнала для части образца из сплава Д16 вокруг отверстия, находящегося под одноосной нагрузкой $57 \mathrm{MPa}$. Размер изображения $0.95 \times 0.95 \mathrm{~mm}$.

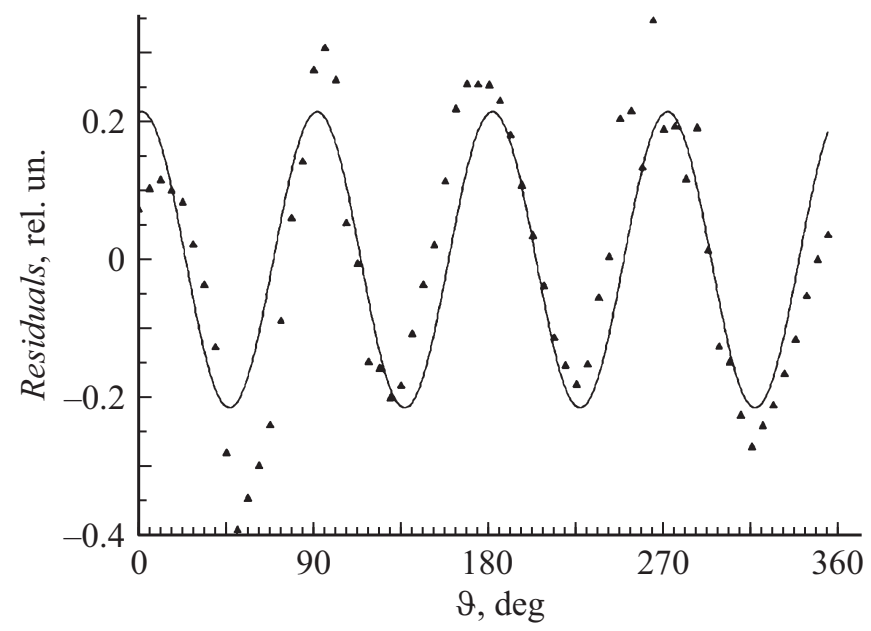

Рис. 2. Нелинейная составляющая ФА-сигнала вдоль окружности с радиусом $0.15 \mathrm{~mm}$. Точки - разность амплитуды ФА-сигнала и его аппроксимации функцией $A_{0}+A_{1} \cos (2 \vartheta)$, кривая - результат подгонки этой разности функцией $A_{2} \cos (4 \vartheta)$.

квадратичных процессов в ФА-сигнал $S$ представим его в виде $S=S_{0}\left(1+b \sigma+c \sigma^{2}\right)$. Тогда в соответствии с равенством (1) ФА-сигнал в полярных координатах будет определяться равенством

$$
S=S_{0}\left(1+A_{1} \cos (2 \vartheta) / r^{2}+A_{2}(1+\cos (4 \vartheta)) / r^{4}\right),
$$

где $A_{1}=2 b \sigma_{x} a^{2}+4 c \sigma_{x}^{2} a^{2}, A_{2}=2 c \sigma_{x}^{2} a^{4}$.

Для визуализации нелинейной составляющей амплитуды ФА-сигнала на основе экспериментальных данных из них вычиталась линейная составляющая, аппроксимированная в соответствии с формулой $S=S_{0}\left(1+A_{1} \cos (2 \vartheta) / r^{2}\right)$. Распределение полученной таким образом нелинейной составляющей ФА-сигнала вблизи отверстия представлено на рис. 1. На нем виден вклад в ФА-сигнал с угловой зависимостью типа $\cos (4 \vartheta)$. На рис. 2 для большей ясности приведена зависимость амплитуды нелинейной компоненты ФА-сигнала от угла для расстояния $150 \mu \mathrm{m}$ от центра отверстия. Отметим, что для этого расстояния амплитуда нелинейной составляющей ФА-сигнала достигала $25 \%$ от линейной компоненты.

На основании полученных экспериментальных данных для поведения ФА-сигнала вблизи отверстия можно оценить значения параметров $A_{1}$ и $A_{2}$. Аппроксимация экспериментальных данных для области $150<r<500 \mu$ m дает следующие значения параметров: $A_{1}=-16960 \pm 30 \mu \mathrm{m}^{2}, \quad A_{2}=(1.54 \pm 0.19) \cdot 10^{9} \mu \mathrm{m}^{4}$. Для отношения коэффициентов $b / c$ получим значение $b / c=-55 \pm 22 \mathrm{MPa.} \mathrm{Отсюда,} \mathrm{полагая} \mathrm{радиус}$ $a=135 \pm 10 \mu \mathrm{m}$ и $\sigma_{x}=57 \pm 3 \mathrm{MPa}$, получаем

$$
\begin{aligned}
S(r, \vartheta) & =S_{0}\left[1-(1.5 \pm 0.4) \cdot 10^{-2} \sigma(r, \vartheta)\right. \\
& \left.+(2.7 \pm 1.1) \cdot 10^{-4} \sigma^{2}(r, \vartheta)\right],
\end{aligned}
$$

где зависимость $\sigma$ от угла и координат определяется равенством (1), а значение $\sigma$ выражается в МРа.

Выражение (3) определяет зависимость ФА-сигнала от напряжения вблизи отверстия для сплава алюминия Д16 и позволяет оценить вклад в него нелинейных процессов. В связи с полученным результатом необходимо обратить внимание на одну важную деталь. Максимальная величина напряжений вблизи отверстия при использованных в наших экспериментах внешних напряжениях не превосходила $180 \mathrm{MPa}$ Оценка дополнительных термоупругих напряжений, создаваемых возбуждающим лазерным излучением, показывает [14], что они находились на уровне $10 \mathrm{MPa}$. Таким образом, общие напряжения, действующие в образце в процессе экспериментов, не выходили за рамки линейной теории упругости данного материала [15].

Для объяснения появления нелинейного вклада в ФА-сигнал от напряжений воспользуемся подходом, предложенным в работах $[10,11]$. В соответствии с ним в уравнении движения для решетки неидеального металла необходимо учитывать дополнительное давление, создаваемое частью электронов при их переходе из связанного состояния в квазисвободное. Для определения количества таких электронов можно воспользоваться уравнением баланса с соответствующим генерационным источником. При чисто тепловом механизме возбуждения электронов такой источник может быть записан в виде [11]:

$$
J_{e}=A\left[\exp \left(-\frac{U}{k\left(T_{0}+\delta T\right)}\right)-\exp \left(-\frac{U}{k T_{0}}\right)\right],
$$

где $U=U_{0}+V \sigma$ - энергия активации электрона, $V-$ активационный объем, $k-$ постоянная Больцмана, 
$T_{0}$ - средняя температура нагретой области, $\delta T-$ переменная составляющая температуры, $A-$ некоторый коэффициент. Считая, что $\delta T \ll T_{0}$ и $V \sigma / k T_{0} \ll 1$, из (4) получим

$$
\begin{aligned}
J_{e} & \cong A \exp \left(-\frac{U_{0}}{k T_{0}}\right)\left(\frac{U_{0}}{k T_{0}}-\frac{U_{0}-k T_{0}}{k T_{0}} \frac{V \sigma}{k T_{0}}\right. \\
& \left.+\frac{U_{0}-2 k T_{0}}{2 k T_{0}}\left(\frac{V \sigma}{k T_{0}}\right)^{2}\right) \frac{\delta T}{T_{0}} .
\end{aligned}
$$

При лазерной генерации ультразвука с гармоническим законом модуляции во времени $\delta T \propto \exp (i \omega t)$ и в соответствии с результатами работы [11] для отношения ФАсигналов $S$ и $S_{0}$ получим следующий результат:

$$
\begin{aligned}
\frac{S}{S_{0}} \cong 1+\frac{2}{3} \frac{1-2 v}{E} \frac{\tau E_{\mathrm{F}} A \exp \left(-\frac{U_{0}}{k T_{0}}\right)}{\alpha T_{0}(1-i \omega \tau)} \\
\\
\times\left[\frac{U_{0}}{k T_{0}}-\frac{U_{0}-k T_{0}}{k T_{0}} \frac{V \sigma}{k T_{0}}+\frac{U_{0}-2 k T_{0}}{2 k T_{0}}\left(\frac{V \sigma}{k T_{0}}\right)^{2}\right],
\end{aligned}
$$

где $\alpha-$ коэффициент теплового расширения, $v-$ коэффициент Пуассона, $E$ - модуль упругости, $E_{\mathrm{F}}-$ энергия Ферми, $\tau$ - время релаксации электрон-решеточной системы, $\omega$ - циклическая частота модуляции лазерного излучения и соответственно частота акустических колебаний.

Полученный результат показывает, что для обеспечения линейности теории термоупугости напряженных металлов помимо обычных механических условий требуется выполнение дополнительного условия $V \sigma / k T_{0} \ll 1$. Выражение (6) позволяет оценить величину активационного объема для сплава Д16. Из него следует соотношение

$$
\frac{b}{c}=-\frac{U_{0}-k T_{0}}{\left(U_{0}-2 k T_{0}\right) V} .
$$

По поводу полученного результата необходимо сделать следующие замечания. Во-первых, следует считать $U_{0}>k T_{0}$, поскольку в противном случае все центры уже в исходном состоянии будут активированы. Во-вторых, исходя из представленных экспериментальных данных отношение $b / c$ для сплава Д16 отрицательно, поэтому в соответствии с равенством (7) в нашем случае должно выполняться неравенство $U_{0}>2 k T_{0}$. Более того, в соответствии с рассматриваемыми физическими процессами $U_{0}$ не может значительно отличаться от $k T_{0}$, и при оценке активационного объема можно считать $U_{0}-k T_{0}$, $U_{0}-2 k T_{0}$. порядка $k T_{0}$. Тогда, используя полученное из эксперимента значение отношения $b / c=-55 \pm 22 \mathrm{MPa}$ и соотношение (7), для активационного объема получим следующую оценку: $V \approx 10^{-21} \mathrm{~cm}^{3}$. Это значение дает оценку для концентрации центров, вносящих вклад в термоупругую генерацию акустических волн в сплаве Д16 при использовавшихся в работе напряжениях.
Таким образом, полученные результаты демонстрируют наличие нового нелинейного фотоакустического эффекта в напряженных металлах при термоупругой генерации звука. Он связан с термоактивационным возбуждением электронной подсистемы и характеризуется новым для термоупругости металлов параметром $V \sigma / k T_{0}$. Особенность данной нелинейности состоит в том, что она может проявляться уже на основной гармонике модуляции лазерного излучения. Полученные результаты хорошо согласуются с выводами работы [16] о необходимости учета вклада электронной подсистемы при рассмотрении деформационных процессов в металлах.

\section{Финансирование работы}

Работа выполнена при поддержке Российского научного фонда в рамках проекта № 15-19-00182.

\section{Конфликт интересов}

Авторы заявляют, что у них нет конфликта интересов.

\section{Список литературы}

[1] Cheng B., Lei J., Xiao H. // Optics Laser Technol. 2019. V. 115. P. 459-464. https://doi.org/10.1016/j.optlastec.2019.02.055

[2] Muratikov K.L., Glazov A.L., Rose D.N., Dumar J.E. // J. Appl. Phys. 2000. V. 88. N 5. P. 2948-2955. https://doi.org/10.1063/1.1287526

[3] Муратиков К.Л., Глазов А.Л., Роуз Д.Н., Думар Д.Е. // Письма в ЖТФ. 2002. Т. 28. В. 9. С. 48-57.

[4] Everton S., Dickens P., Tuck C., Dutton B. // JOM. 2018. V. 70. N 3. P. $378-383$. https://doi.org/10.1007/s11837-017-2661-7

[5] Selim H., Prieto M.D., Trull J., Romeral L., Cojocaru C. // Sensors. 2019. V. 19. N 3. P. 573 (1-12). https://doi.org/10.3390/s19030573

[6] Муратиков К.Л., Глазов А.Л., Николаев В.И. // Письма в ЖТФ. 2005. Т. 31. В. 7. С. 19-25.

[7] Глазов А.Л., Морозов Н.Ф., Муратиков К.Л. // ФТТ. 2016. T. 58. B. 9. C. $1679-1687$.

[8] Nowacki $W$. Thermoelasticity. Oxford: Pergamon Press, 1986. $578 \mathrm{p}$.

[9] Ландау Л.Д., Лифиии Е.M. Теория упругости. М.: Наука, 1987. $246 \mathrm{c}$.

[10] Морозов Н.Ф., Муратиков К.Л., Семенов Б.Н., Индейцев Д.А., Вавилов Д.С. // ДАН. 2019. Т. 485. № 4. С. 438 441.

[11] Глазов А.Л., Муратиков К.Л. // Письма в ЖТФ. 2019. Т. 45. B. 17. C. 51-54.

[12] Биргер И.А. Остаточные напряжения. М.: МАШГИЗ, 1963. $232 \mathrm{c}$.

[13] Тимошенко С.П., Гудьер Джс. Теория упругости. М.: Наука, 1979. $560 \mathrm{c}$. 
[14] Муратиков К.Л. // ЖТФ. 2011. Т. 81. В. 2. С. 58-63.

[15] Грищенко Н.А., Сидельников С.Б., Губанов И.Ю., Лопатина Е.С., Галлиев Р.И. Механические свойства алюминиевых сплавов. Красноярск: СФУ, 2012. 196 с.

[16] Зуев Л.Б., Данилов В.М., Плосков Н.А. // Письма в ЖТФ. 2018. T. 44. В. 13. C. $75-79$.

https://doi.org/10.21883/PJTF.2018.13.46330.17088 